\title{
Correction to: Orbit insertion strategy of Hayabusa2's rover with large release uncertainty around the asteroid Ryugu
}

Yusuke Oki ${ }^{1}(\otimes)$, Kent Yoshikawa ${ }^{2}$, Hiroshi Takeuchi ${ }^{2}$, Shota Kikuchi ${ }^{2}$, Hitosi Ikeda ${ }^{2}$, Daniel J. Scheeres ${ }^{3}$, Jay W. McMahon ${ }^{3}$, Junichiro Kawaguchi ${ }^{2}$, Yuto Takei ${ }^{2}$, Yuya Mimasu ${ }^{2}$, Naoko Ogawa $^{2}$, Go Ono ${ }^{2}$, Fuyuto Terui ${ }^{2}$, Manabu Yamada ${ }^{4}$, Toru Kouyama ${ }^{5}$, Shingo Kameda ${ }^{6}$, Kazuya Yoshida $^{7}$, Kenji Nagaoka ${ }^{8}$, Tetsuo Yoshimitsu ${ }^{2}$, Takanao Saiki ${ }^{2}$, and Yuichi Tsuda ${ }^{2}$

1. Japan Aerospace Exploration Agency, Tsukuba 305-8505, Japan

2. Japan Aerospace Exploration Agency, Sagamihara 252-5210, Japan

3. University of Colorado, Boulder, CO 80309, USA

4. Planetary Exploration Research Center, Chiba Institute of Technology, Narashino 275-0016, Japan

5. National Institute of Advanced Industrial Science and Technology, Koto-ku 135-0064, Japan

6. Rikkyo University, Toshima-ku 171-8501, Japan

7. Department of Aerospace Engineering, Tohoku University, Sendai 980-8579, Japan

8. Kyushu Institute of Technology, Kitakyusyu 804-8550, Japan

(C) The Author(s) 2020, Corrected Publication January 2021

Correction to:

Oki, Y., Yoshikawa, K., Takeuchi, H. et al.

Orbit insertion strategy of Hayabusa2's rover with large release uncertainty around the asteroid Ryugu.

Astrodynamics 2020, 4(4): 309-329

https://doi.org/10.1007/s42064-020-0080-y

The article "Orbit insertion strategy of Hayabusa2's rover with large release uncertainty around the asteroid Ryugu" written by Yusuke Oki, Kent Yoshikawa, Hiroshi Takeuchi et al., was originally published electronically on the publisher's internet portal (currently SpringerLink) on 05 November 2020 without open access. After publication in Volume 4, Issue 4, page 309-329, the author(s) decided to opt for Open Choice and to make the article an open access publication. Therefore, the copyright of the article has been changed to $($ The Author(s) 2020 and the article is forthwith distributed under the terms of the Creative Commons Attribution 4.0 International License (http: //creativecommons.org/licenses/by/4.0/), which permits use, duplication, adaptation, distribution and reproduction in any medium or format, as long as you give appropriate credit to the original author(s) and the source, provide a link to the Creative Commons license, and indicate if changes were made.

The original article has been corrected and can be found at https://doi.org/10.1007/s42064-020-0080-y.

凶 oki.yusuke@jaxa.jp 